\title{
Residue detection for real-time removal of paint from metallic surfaces
}

\author{
Yoseph Bar-Cohen, Xiaoqi Bao, Benjamin Dolgin, and Neville Marzwell \\ Jet Propulsion Laboratory (JPL)/Caltech ${ }^{1}$
}

\begin{abstract}
Paint stripping from large steel ships and other metallic surfaces is a major environmental safety, cost, and operational challenge in effectively and efficiently maintaining and refurbishing large structures. Environmental concerns are greatly limiting the possible options. As a result, a hybrid system composed of a waterjet with water recycling and robotic mobile manipulators with scanning bridges has become the leading form of paint stripping and was constructed by various manufacturers to address this need. The application of such scanning bridges is slow and their access is constrained by the complex shape of the ship hull and various features on the surface. To overcome these limitations, a robotic system that is called UltraStrip (UltraStrip Systems, Inc., Stuart, FL) is developed. This system uses magnetic wheels to attach the stripper to the structure and travel on it while performing paint stripping. To assure efficient paint stripping feedback data is required to control the travel speed by monitoring the paint thickness before and during the stripping process. Efforts at JPL are currently underway to develop the required feedback capability to assure effective paint stripping. Various possible sensors were considered and issues that can affect the sensitivity, reliability and applicability of the sensors are being investigated with emphasis on measuring the initial conditions of the paint. Issues that affect the sensory data in dynamic conditions are addressed while providing realtime real feedback for the control of the paint stripper speed of travel.
\end{abstract}

Keywords: NDE, NDT, Paint, Real-Time Testing, Sensors, Robotics, Marine, Paint Stripping, Waterjet Feedback

\section{Introduction}

The ship hull of marine structures requires a periodic maintenance that involves paint striping and repainting. Current systems employ scanning bridges that are cumbersome, heavy, costly and difficult to operate and to maneuver. Environmental and economical concerns are driving the evolution of a new generation of robots that can travel on the ship hulls and remove the paint using highly pressurized waterjet and a recycling system filtering the removed paint. Since most ship hulls are made of ferromagnetic structure the crawler robots is made to maintain adherence to the surface using magnetic wheels that allow the crawler to operate on vertical surfaces and in upside-down operations [Bar-Cohen and Joffe, 1997]. The magnetic attraction is sufficiently strong to attach the crawler while applying the waterjet is pressurized at a level of $30-40 \mathrm{Ksi}$ and the crawler carries wiring cables and water hoses. A photographic view of the robotic system called UltraStrip [http://www.jpl.nasa.gov/pictures/tech/paintstripper.html and http://www.ultrastrip.com], which is the objective of the authors study, is shown in Figure 1.

FIGURE 1: The waterjet paint remover robot, UltraStrip, is shown in operation on a ship hull surface (the removed paint on the right).

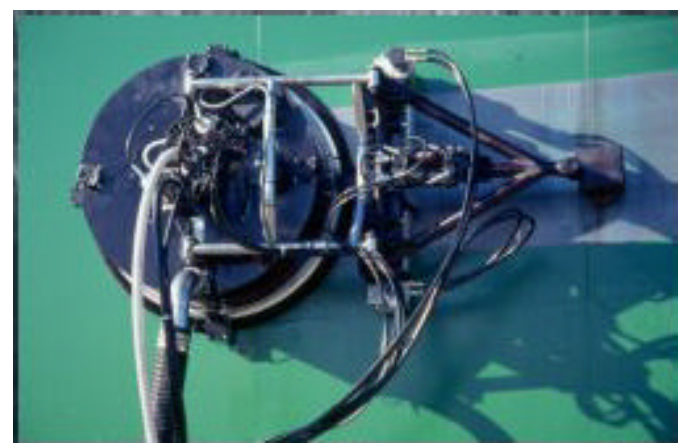

Currently, operators of the paint-stripping crawler are choosing the travel speed based on a visual examination of the area that was covered by the crawler and the level of paint residues. In order to automate this process while optimizing the stripping performance, which might have to handle multilayers of paint of various thicknesses, it is necessary to use sensors to provide feedback about the stripped paint and the thickness of the residual paint. The JPL team in cooperation with researchers at the NASA - National Engineering Robotics Consortium (NREC) at Carnegie Mellon University, PA,

${ }^{1}$ (MC 82-105), 4800 Oak Grove Drive, Pasadena, CA 91109-8099, Phone 818-354-2610, Fax 818-393-3254, yosi@.jpl.nasa.gov web: http://ndea.jpl.nasa.gov 
investigated various possible sensors towards developing a real-time gauging system to provide feedback to the crawler. The sensors that were considered needed to address two objectives: (a) Gauge the paint thickness to determine the need to continue the waterjet action; and (b) Identify presence of paint in go/no-go form within user-defined tolerances to be sure that the paint was fully removed. Three types of sensors were considered in this study including electromagnetic proximity sensor, ultrasonic transducer and conductivity finger array. The results of this study are reported in this manuscript.

\section{Quantitative Thickness Gauging}

Depending on the specific user requirements, there are several gauging techniques that can be used to provide feedback to a paints stripping crawler and they can be operated either selectively or in a combined form. To obtain quantitative data eddy current, magnetic and ultrasonic sensors were investigated whereas conductivity gauging was studied for go/on-go considerations.

\subsection{Eddy Current Sensor}

Generally, eddy current offers a widely used method of gauging the thickness of paint. Using eddy-current gauging provides sensitivities and accuracies at the micron levels however difficulties are encountered when the stripper travels and it is difficult to assure fixed distance of the gauge to the paint surface and that critically impacts the measurement accuracy. The major concern regarding the use of proximity sensors is the fact that they gauge the distance of the probe to the metal surface rather than directly gauging the thickness of the paint.

This method involves inducing circular electric currents in conductive objects that are placed adjacent to a coil with AC current. These currents are the result of the reaction to the effect of the electromagnetic field on the free electrons in the conductor. The eddy current creates an opposite magnetic field, which partially cancels the magnetic flux through the coil and, then, reduces the inductance of the coil. This phenomenon is sensitive to the coil liftoff and material conductivity. The variations in the conductivity can be minimized by pre-calibration. After proper calibration of the effect of liftoff one can determine the thickness of coatings on conductive materials. The use of high frequencies can take the advantage of the skin effect associated with the eddy current phenomenon. The higher the frequency that is used the shallower the electromagnetic field is penetrated. The use of high frequencies reduces the effect of the thickness of the conductive material.

Many eddy current based commercial instruments are available for gauging coatings on aluminum surfaces. The output of the in strument is the amplified voltage of an impedance bridge. An example of the output voltages versus liftoff distances is presented in Figure 2 for operating frequency of $1 \mathrm{MHz}$. The sensitivity varies from $1000 \mathrm{mV} / \mathrm{mm}$ to 100 $\mathrm{mV} / \mathrm{mm}$ in the range from 0 to $3 \mathrm{~mm}$ and the noise level is about $0.1 \mathrm{mV}$ and the related resolution is 0.1 to $1 \mu \mathrm{m}$ in this range. Actual accuracy depends on the surface conditions (curvature, roughness), perpendicularity of the sensor, etc.

FIGURE 2: Eddy current sensor on aluminum plate

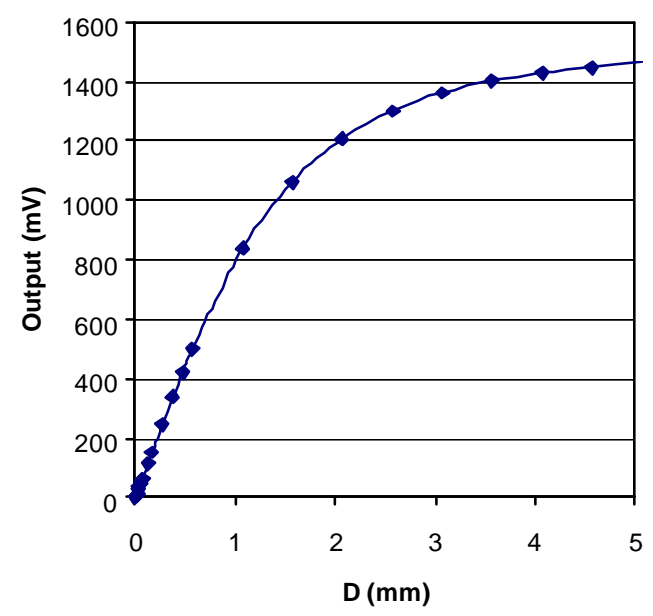

Using the same sensor to the liftoff for a steel plate involves with a different response due to the ferromagnetic nature of steel. Besides the eddy current phenomenon, which decreases the inductance of the coil, the high magnetic permeability significantly increases the inductance. As shown in Figure 3, the output voltage is decreased with the increase in distance from the surface, i.e., liftoff. This implies that the ferromagnetic effect of the steel is much stronger and dominant for the tested sensor. The output voltage is saturated at a distance range of les s than $0.3 \mathrm{~mm}$ due to the limited dynamic range of the amplifier. The sensitivity is higher and the workable range is narrower than for the aluminum 
plate. In the distance range from 0.3 to $2 \mathrm{~mm}$ the sensitivity varies from 6000 to $100 \mathrm{mV} / \mathrm{mm}$, respectively. Our tests indicated a serious output shift due to remnant magnetic field on the steel plate caused by the magnetic wheels of the crawler. Although a strong DC magnetic field induced to cause magnetic saturation of the material may avoid the variations associated with the remnant field, the device will increase the cost and complexity of the sensor. We concluded that the eddy current sensor is suitable for monitoring paint thickness on non-ferromagnetic metallic plate but may not be a good choice for a crawler with magnetic wheels on ferromagnetic plates.

FIGURE 3: Eddy current sensor on steel plate

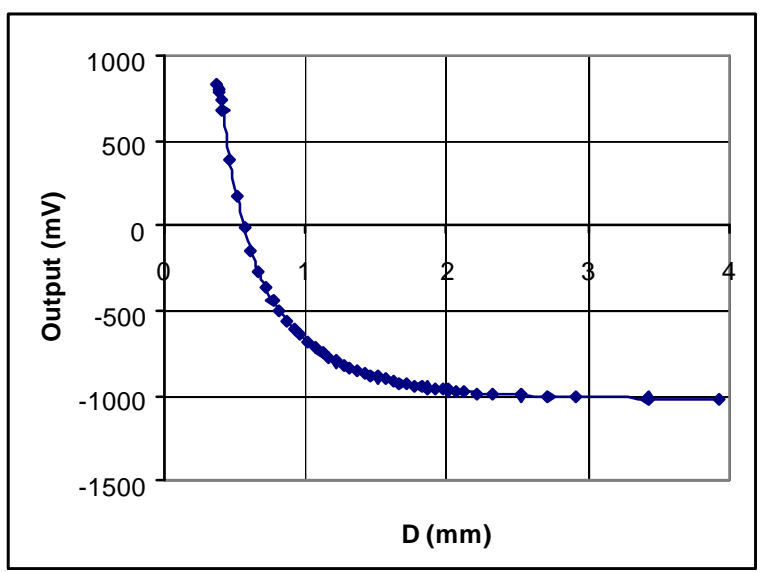

\subsection{Magnetic thickness sensor}

Magnetic sensors are used for thickness gauging tasks where a coil is operated on the principle of a transformer. It consists of two coupled coils, primary and secondary (Figure 4). The ferromagnetic substrate strengthens the inductive coupling between the two coils and increases the output from the secondary coil, which is the measured signal. The signal is sensitive to the distance of the sensor from the ferromagnetic substrate. The operating frequency is relatively low, in the hundreds $\mathrm{Hz}$ range, and it is selected low to avoid the influence of the eddy current phenomenon.

FIGURE 4: Principle of magnetic sensor

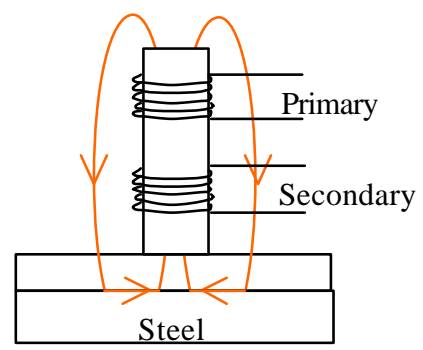

The authors used a magnetic sensor Elcometer Model 345/F3 (Elcometer, Inc.) and tested its response using a steel substrate. The results are shown in Figure 5. The readings were robust and no significant variations were found on the traces of magnetic wheels and the measured error is shown graphically in Figure 6 . The accuracy is $3 \%+0.05 \mathrm{~mm}$ in the range of $0-8 \mathrm{~mm}$ and may be improved by careful calibrations. 


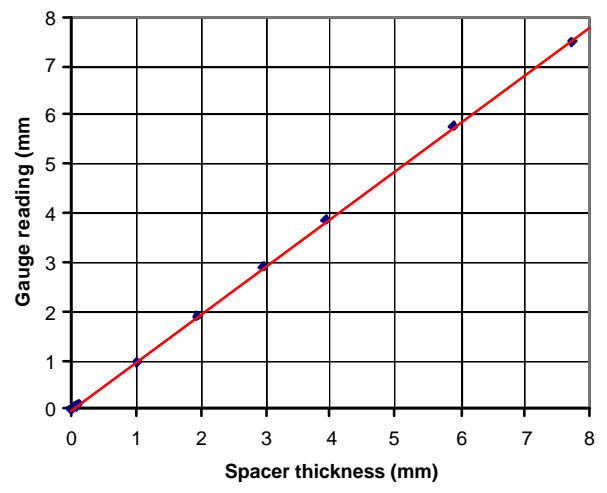

FIGURE 5: Magnetic thickness sensor on steel plate

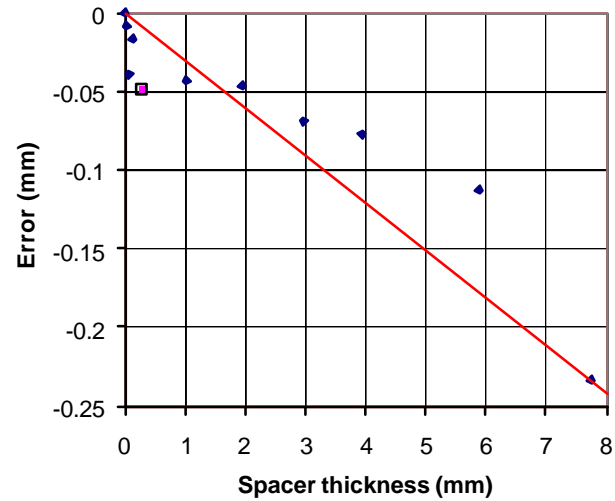

FIGURE 6: Errors of the magnetic sensor

\subsection{Ultrasonic gauging}

The use of ultrasonic thickness gauging sensor is ideal since such test requires use of water coupling medium that is available as part of the waterjet stripping system. Using transducers at frequencies in the range of $1-10 \mathrm{MHz}$ can provide the needed thickness information by specifically gauging the paint thickness rather than obtaining indirect information that results from the liftoff effect as in eddy current and the magnetic testing. Accommodations for the effect of the stripped paint and air bubbles need to be provided to assure the accuracy of the ultrasonic measurements. Use of rapid spectral analysis allows reducing the effect of noise and unnecessary interferences on the measurements. When using ultrasonic thickness gauging in pulse-echo mode the pulses travel thru the paint are reflected from the paint interfaces with the water medium on the front surface and with the metal substrate on the rear. The accuracy of this measurement depends on the uniformity of the wave velocity and the attenuation of the wave in the paint. Generally, thickness values of microns to meters can be measured using this technique and the data can be acquired in real time. Figures 7 shows the waveforms of reflections from a 1.4-mm thick paint applied on a 15-mm thick steel plate. The first reflection is obtained from the top surface of the paint and the second from its bottom. Higher frequencies offer higher resolution and ability to measure smaller thickness values however higher attenuation is encountered limiting the maximum measurable range. A trade off in frequency selection may be required to obtain required to simultaneously optimize the selection of the resolution and frequency range.

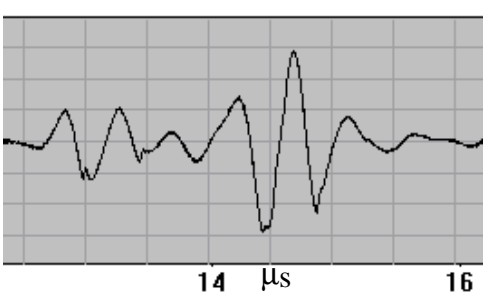

$\mathrm{f}=2.25 \mathrm{MH}$

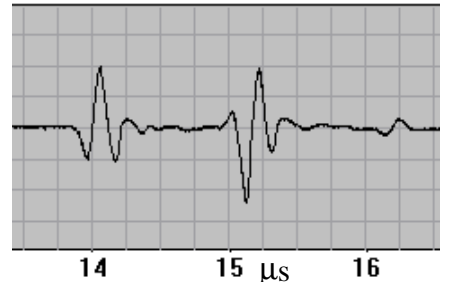

$\mathrm{f}=5 \mathrm{MHz}$

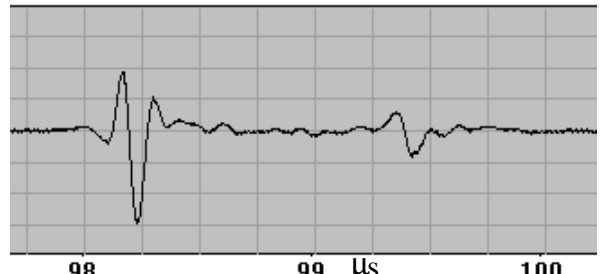

98

$\mathrm{f}=10 \mathrm{MHz}$

Figure 7: Reflections from a painted surface (paint thickness is $1.4 \mathrm{~mm}$ ) at various frequencies.

\subsection{Comb electrode array}

\section{Qualitative Thickness Gauging}

This sensor consists of conductive fingers that are attached to the tested surface and the conductivity is measured. It is assumed that the conductivity of the paint is significantly lower than the metal of the ship-hull. This sensor offers fast feedback regarding the overall paint stripping process but issues related to the sensitivity, resolution, and reliability need to be addressed.

A set of comb electrode array was designed to confirm the total removal of paint from the surface. The orientation of the finger array is set perpendicular to the direction of motion trailing behind the waterjet column. Data acquired from the sensors provides a two-dimensional map of the distribution of paint residues. The number of elements in the array and the data reading speed for each of the individual electrodes are determined by the desired resolution. The number of wires that are used can reach hundreds depending on the required resolution and width of the scanned area. This sensor 
is shown schematically in Figure 7 and it consists of multiple contact wires that scan the surface to provide conductivity image of the test area. For reliable and long lifetime operation, the wires are made of metal that is elastic, corrosionresistant, and has a good wear-resistance.

FIGURE 7: Comb sensor provides assurance of the paint removal.

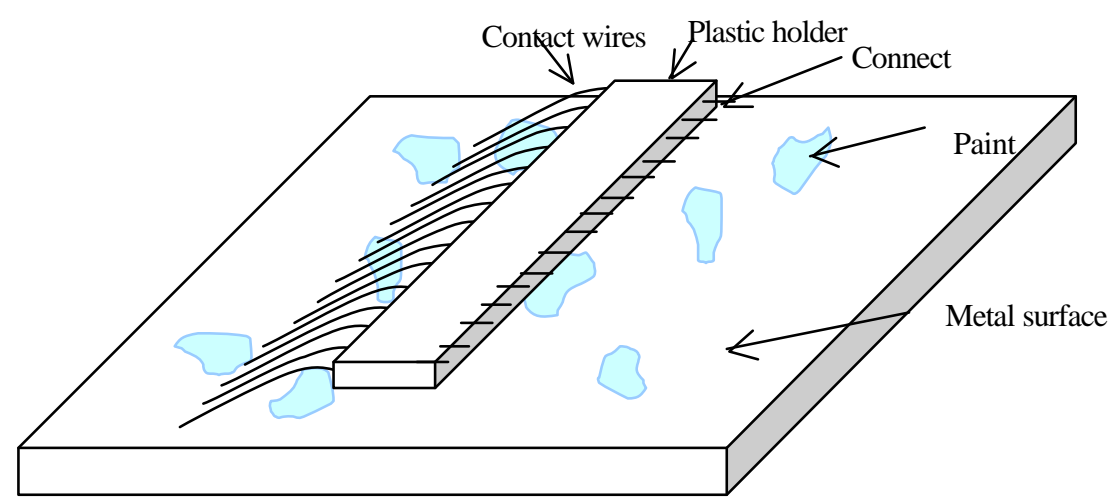

The data acquisition from the comb wires needs to be multiplexed to obtain input from each wire using minimum amount of hardware. This data is used to produce a map of the condition of the stripped paint (Figure 8). The multiplexer is controlled by a microprocessor/computer onboard the robotic crawler. The microprocessor/computer processes the output from the multiplexer and produces a 2-D image. Also, it provides control parameters for the waterjet mechanism to support making decisions regarding corrective action or instructions to proceed with the cleaning process.

An array with 40 wires was fabricated and tested on a steel substrate with paints having various simple geometrical shapes. The space between the wires was set as $12.5-\mathrm{mm}$ (0.5 inch). An instrument Multimeter/Data Acquisition System, Keithley 2700, was used as the multiplexer. A computer with control and display software controlled the instrument and displayed on the screen the conductive pattern of the tested paint. Figure 8 shows the image of an area that has the size of $20 \times 30 \mathrm{~cm}^{2}$.

FIGURE 8: Control system using the wire comb sensor array.

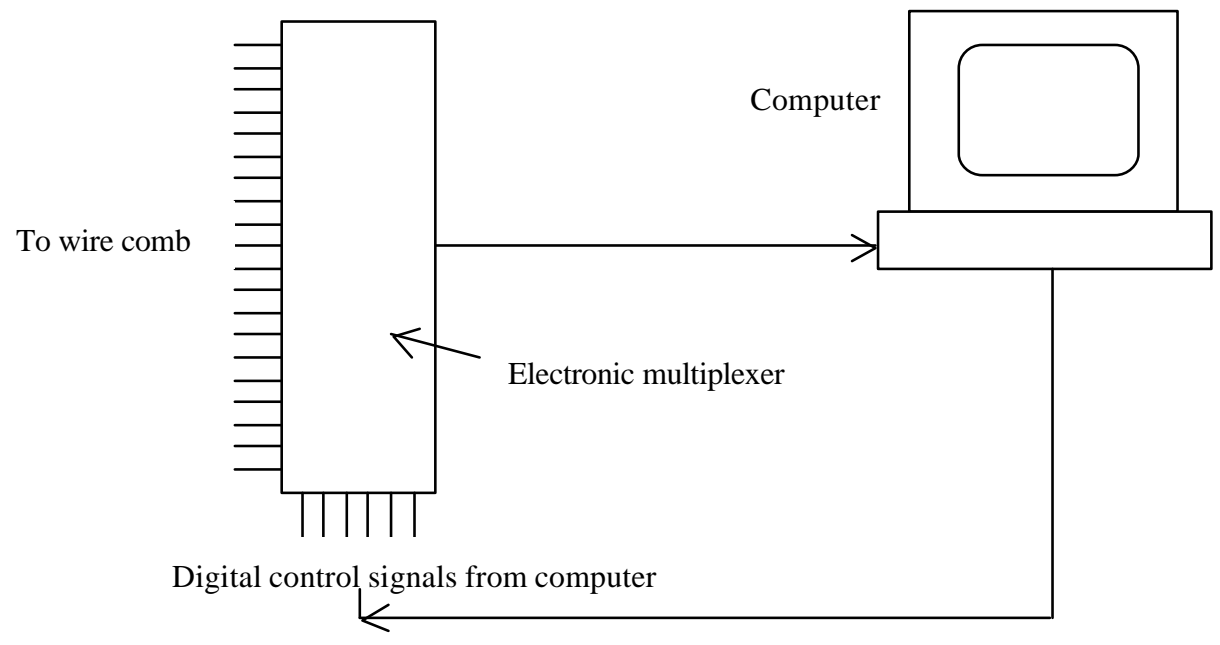

FIGURE 9: Conduct image of the sample plate

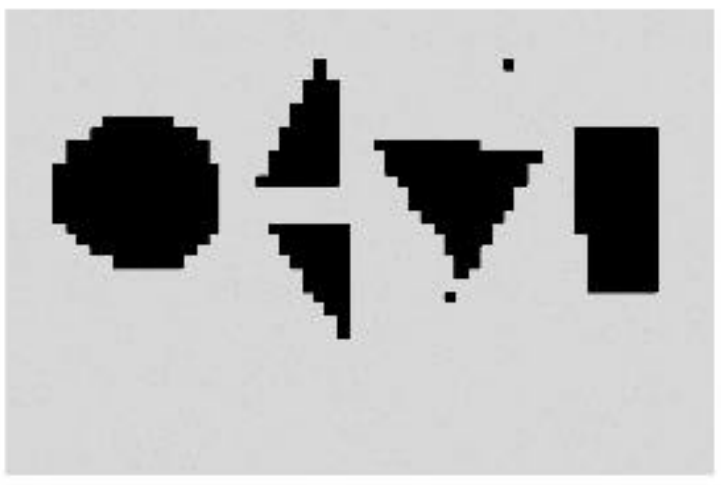




\section{Ultrasonic Paint Loosening}

The process of paint removal using waterjet can be enhanced using high power ultrasonics [Bar-Cohen, Bao and Marzwell, 2000]. Generally, ultrasonic waves traveling in water generate several phenomena that can be harnessed to loosen paint. These phenomena include ability to focus and concentrate energy at the desired location, generate cavitation with strongly destructive power, raise the temperature, and cause water streaming [Brown and Goodman, 1965]. These phenomena provide an enormous capability if optimized to operate with the waterjet paint-stripper. A photographic view of the field of a high intensity focused ultrasonic transducer that is activated in water at a frequency of about $500-\mathrm{KHz}$ is shown in Figure 10.

FIGURE 10: A view of the acoustic field of a focus transducer driven with high intensity.

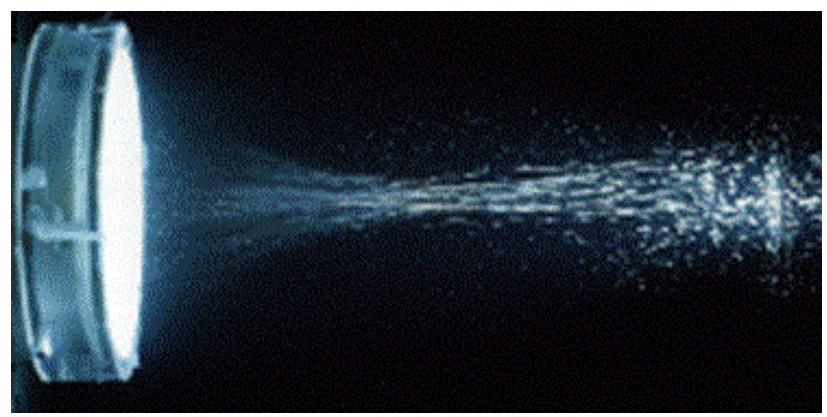

To demonstrate the ability to loosen paint via ultrasound $10-\mathrm{cm}$ diameter focused transducer with $10-\mathrm{cm}$ focal length was driven at $835-\mathrm{KHz}$ focusing the wave onto the painted surface. The sample was made of a $10-$ $\mathrm{mm}$ thick steel plate that had a 3 weeks old 1-mm thick spray-painted layer. Since the test was far from optimal the sample was exposed for a period of 3-hours to cause sufficiently extensive damage at a level that can be seen visually by a necked eye. The induced damage covered an area of about 1-cm diameter and two angle views of the sample are shown in Figure 11. Two important features, which are very important for waterjet stripping, were seen in the exposed area:

- $\quad$ Paint blistering - This damage is caused by two effects of the high intensity ultrasound:

a. Induced high temperature at the focal zone potentially reaching hundreds of degrees.

b. Tensile and shear forces operating on the interface between the steel and the paint. The action of these forces is enhanced by the large acoustic impedance mismatch between steel and the polymeric base paint.

- Focused paint damage - This damage results from the action of induced cavitation.

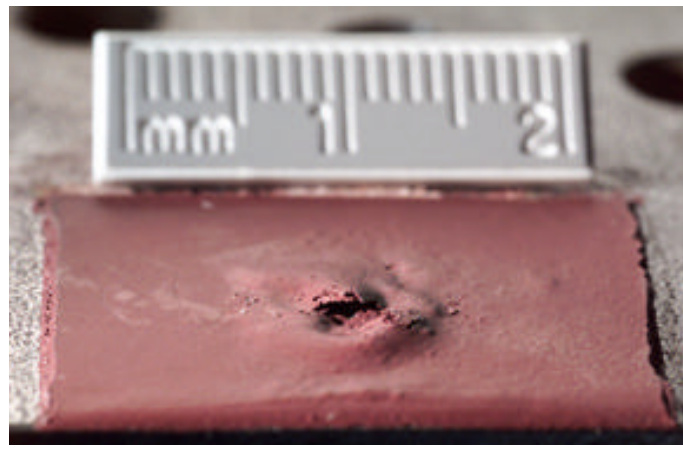

FIGURE 11: A segment of $2.5 \times 2.5 \mathrm{~cm}$ paint subjected to high intensity ultrasound. The paint suffered blistering and physical damage preparing it for easy removal by a waterjet.

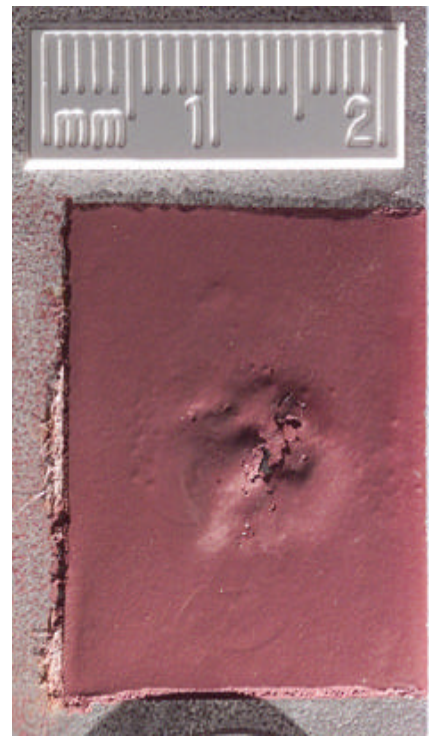

\section{SUMMARY}

A crawler called UltraStrip was recently developed to automatically remove paint from ship hulls that are made of ferroelectric material. To optimize the travel speed of the crawler sensors for real time data acquisition were explored including magnetic, eddy-current, ultrasonic and conductive finger array. The sensors provide quantitative feedback for the control of the crawler travel rate whereas the finger array provides a qualitative map of the paint residues as a go/nogo gauging of the stripped paint. To enhance the paint stripping process a high power focused ultrasonic method was developed to loosen the paint and allow faster stripping by the waterjet. The technology is expected to operate in 
conjunction with wireless communication to support the extensive requirements for data manipulation and system control during the operation of the crawler on ship hulls.

\section{ACKNOWLEDGEMENT}

The research at Jet Propulsion Laboratory (JPL), California Institute of Technology, was carried out under a contract with National Aeronautics Space Agency (NASA) and with the National Engineering Robotics Consortium (NREC), Carnegie Mellon University, PA. The authors would like to thank Dr. Bill Ross, NREC, CMU for his helpful technical comments and suggestions.

\section{REFERENCES}

Bar-Cohen Y., and B. Joffe, "Magnetically Attached Multifunction Maintenance Rover (MAGMER)," Submitted as a New Technology Report, February 6, 1997, Item No. 9854, Docket 20229, August 4, 1997, U.S. Provisional Patent CIT 2680

Bar-Cohen Y., X. Bao and N. Marzwell, "Ultrasonic Paint Loosening and RearTime Thickness Gauging (UPL-RTG) System for enhancement of Waterjet Paint Stripping," Su bmitted May 2, 2000. Docket No. 21063, Item No. 0664B, May 8, 2000. Provisional Patent CIT 3258-p filed on Aug. 14, 2000 (Ref. 06618/684P01).

Brown, B and Goodman, J.E., High-Intensity Ultrasonics: Industrial Applications, Iliffe Books, Ltd., London (1965). 